\title{
PERFIL BIOANTROPOLÓGICO DE LA POBLACIÓN DE IGLESIA COLORADA, VALLE DE COPIAPÓ, EN ÉPOCA INCA.
}

\author{
Catalina Morales ${ }^{1}$ y Francisco Garrido ${ }^{2}$ \\ 'Investigador externo Museo Nacional Historia Natural de Chile, cmoralescifuentes@gmail.com \\ ${ }^{2}$ Curador Área Arqueología, Museo Nacional de Historia Natural de Chile, francisco.garrido@mnhn.gob.cl
}

\section{RESUMEN}

Este artículo presenta los resultados del análisis de los restos humanos del período Tardío recuperados en el sitio Iglesia Colorada, ubicado en el curso alto del valle del Río Copiapó. El objetivo principal es el identificar indicadores de modo de vida y actividad de la población del cementerio, mediante el análisis de su perfil demográfico, indicadores dentales, patologías articulares, alteraciones en la morfología del hueso, e indicadores de estrés fisiológico. Si bien el número de individuos no es suficiente para generar estimaciones estadísticas, se observa una distribución normal en las edades de muerte, una salud oral particularmente buena y escasos indicadores de estrés fisiológico. Pese a haber pocos individuos donde se pudo observar las superficies articulares, estas muestran estrés mecánico principalmente asociado a la carga de peso, a lo que se suman dos casos de alteraciones atípicas en clavículas. Finalmente se discute la presencia de algunas variaciones anatómicas que comúnmente se han interpretado como rasgos discretos, pero que podrían ser el resultado de actividades laborales.

Palabras clave: Bioarqueología, Antropología Biológica, Arqueología, Inca, Copiapó, Paleopatología, Artropatías.

\section{ABSTRACT}

Bioanthropological profile of the population of Iglesia Colorada, Copiapó valley, in Inca times. This paper presents the results of human remains analysis from Iglesia Colorada site, located in the upper course of Copiapó valley. Our main objective is to identify the way of life and activities of the people buried at the cemetery by analysing their demographic profile, dental indicators, joint disorders, alterations in bone morphology and physiological stress indicators. Although the sample size is not enough to generate confident statistical interpretations, we observed a normal distribution of age of death, good oral health, and scarce physiological stress indicators. Although we could observe the joint surfaces of a small number of individuals, they show mechanical stress associated with heavy load carrying, including two atypical alterations in the clavicle. Finally, we discuss the presence of some anatomical variations that commonly have been interpreted as discrete traits but could be the results of work-related activities.

Key words: Bioarcheology, Biological Anthropology, Archeology, Inca, Copiapó, Paleopathology, Arthropathies.

\section{INTRODUCCIÓN}

\section{CONTEXTO DEL SITIO IGLESIA COLORADA}

Durante el Periodo Intermedio Tardío (1200 d.C. - 1400 d.C.), la población local del valle de Copiapó corresponde a la denominada "Cultura Copiapó", sobre la cual llegaría la imposición del estado Inca a principios del siglo XV. La Cultura Copiapó estaría representada por los estilos cerámicos "Copiapó Negro sobre Rojo", "Copiapó Negro sobre Rojo y Crema" y "Punta Brava" (Garrido 2014). El conocimiento sobre la estructura social de este grupo en época preinca es escaso, ya que pocos asentamientos han sido excavados y la mayor parte de ellos posee en mayor o menor medida una ocupación del período Tardío. De todos modos, es evidente que esta fue una sociedad sin una jerarquía importante de asentamientos, presentándose estos como pequeños poblados dispersos en el valle (Castillo 1998; Iribarren 1958). Es posible que la relación entre la Cultura Copiapó y el imperio Inca haya sido mediada por la presencia 
Diaguita. Esta interpretación se basa en la presencia de estilos cerámicos Diaguita III con influencia Inca, sugiriendo una expansión imperial de sur a norte hacia el valle de Copiapó (Berenguer 2009; Cornejo 2001; González 2013; Troncoso y Pavlovic 2013; Uribe y Sánchez 2016).

Durante la época de ocupación incaica en Copiapó, se ha observado un cambio en el uso del territorio y la explotación de los recursos. Se reconoce un aumento en la ocupación de sitios en vegas y cursos de agua de la porción alta de la cuenca, especialmente en los afluentes del río Copiapó, como son el río Pulido y el río Jorquera. Destacan centros administrativos, tambos, adoratorios de altura como el Volcán Jotabeche y el Volcán Copiapó, la fundición de Viña del Cerro, y el poblado de Iglesia Colorada (Castillo, 1998; Niemeyer, 1986, 1993). De acuerdo a Niemeyer toda la infraestructura incaica tanto administrativa como productiva habría estado en función de la minería, siendo esta la "única producción excedentaria y exportable" (Niemeyer 1986:219). Evidencia de aquello es la fundición de cobre de Viña del Cerro, un centro de producción metalúrgica Inca formado por un patrón arquitectónico de recintos rectangulares, una plaza, una posible plataforma ceremonial o ushnu y 26 unidades de hornos de fundición (posiblemente del tipo "huayras"). Si bien es posible que la escala de producción de Viña del Cerro en época Inca no haya sido tan grande como se pensaba anteriormente, lo relevante es que incluso sitios de esta envergadura son parte de una lógica local previa de trabajo minero-metalúrgico y tienen una ocupación con un origen preincaico (Garrido y Plaza 2020). Además, este tipo de producción metalúrgica coexistió con una producción local de lapidaria y pigmentos, representada por sistemas mineros como el de la sierra Cachiyuyo de Llampos que, sin haber estado bajo el control de la economía política Inca, aprovecharía la infraestructura imperial de caminos y tambos para fortalecer un desarrollo económico local (Garrido 2016; Garrido y Salazar 2017).

Este es el contexto en el que se inserta el sitio arqueológico Iglesia Colorada, el cual es un importante centro administrativo Inca situado en la cuenca alta del río Copiapó (Castillo, 1998; Niemeyer 1986). Este sitio se emplaza a $1.580 \mathrm{msnm}$ a la izquierda del río Pulido, y a unos $120 \mathrm{~km}$ de la ciudad de Copiapó. Fue investigado por Hans Niemeyer desde la década de 1960 hasta el año 2003, y es reconocido como uno de los principales núcleos de asentamiento incaico en el valle, interviniendo una aldea local (Castillo 1998). El sitio se compone de 3 sectores; a) Potrero El Damasco, b) Potrero Las Tamberías y c) Fundo Bauzá.

El sector Potrero El Damasco presenta grandes estructuras arquitectónicas de planta rectangular y muro de doble hilada de estilo incaico, además de un espacio funerario identificado en las excavaciones de 1994-1995. En dicha campaña se excavaron nueve sepulturas de características similares, más una décima diferente del resto, cuyo ajuar y contexto corresponde al periodo Intermedio Tardío (Castillo 1998; Garrido y Morales 2019). El patrón de entierro se caracteriza por fosas abovedadas cuadradas o rectangulares, sin señalización superficial, algunas con piedras laja de cubierta, un número variable de escalones, y en algunos casos con un techo de palos de algarrobo. En el año 2003 se realizó una nueva excavación en el área funeraria del Potrero El Damasco, la cual registró cuatro nuevas sepulturas. Cuatro de los individuos recuperados están representados únicamente por sus cráneos y algunas vértebras cervicales. Estos cráneos presentan perforaciones, evidencias de descarnamiento y exhibición, lo que evidencia prácticas de violencia ideológica por parte del imperio Inca hacia las poblaciones locales (Garrido y Morales 2019). En total se registraron 16 sepulturas en el sector El Damasco, cuyos fechados radiocarbónicos están entre el 1400-1500 d.C., excepto por la sepultura 10 cuyo fechado y cultura material la ubicaría en el periodo Intermedio Tardío (Figura 1).

El segundo sector del sitio corresponde a Potrero Las Tamberías, a unos 300 metros de Potrero El Damasco. Este se compone de un conjunto de estructuras residenciales del mismo tipo arquitectónico incaico. Aquí no se han hallado hasta ahora sepulturas. Finalmente, el tercer sector corresponde al Fundo Bauzá, el cual se compone de un área funeraria descubierta durante la reparación de un camino en 2016, en donde se identificaron dos sepulturas con características similares a las registradas en El Damasco, conteniendo los restos humanos de cuatro individuos. 


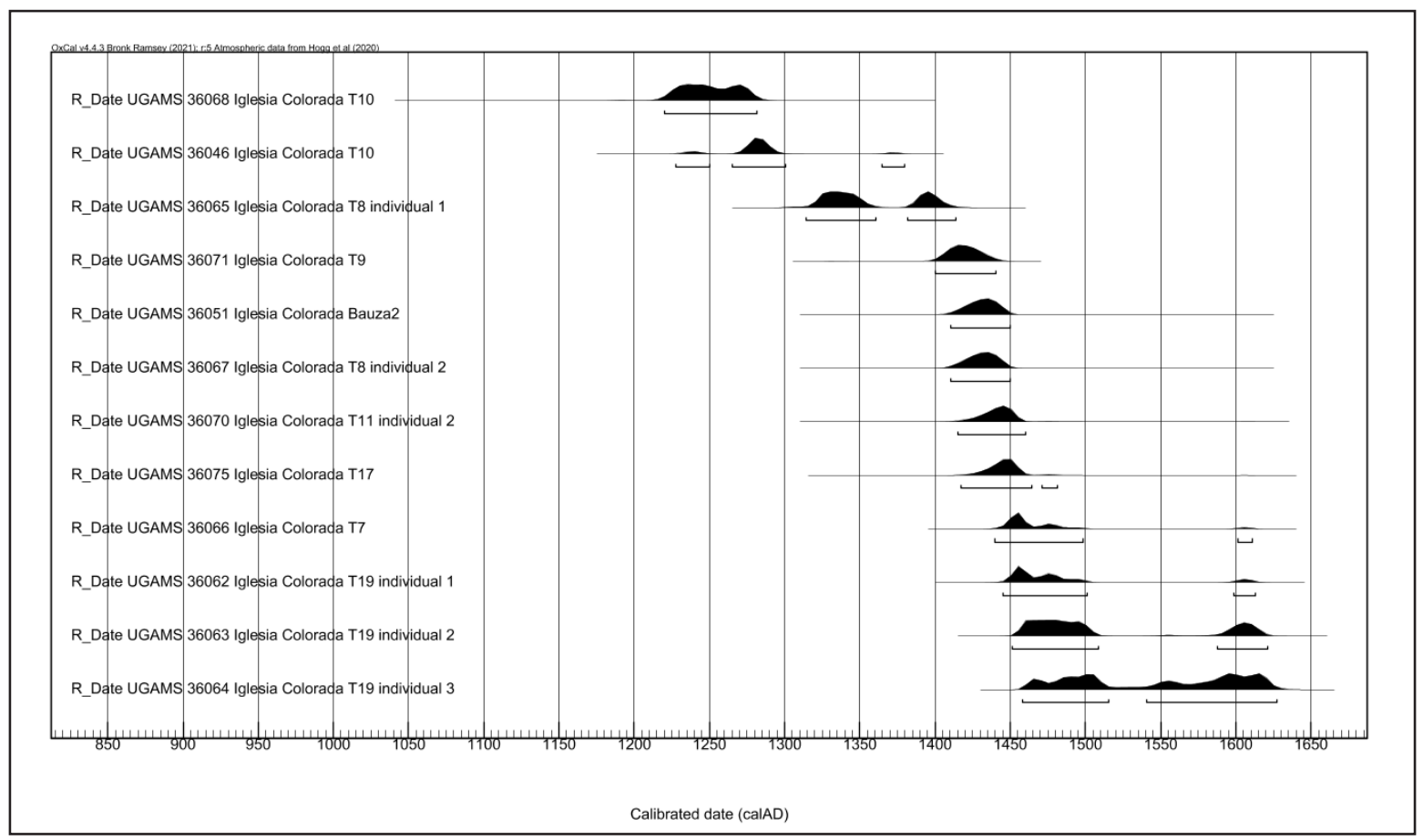

Figura 1: Fechados radiocarbónicos de los individuos de Iglesia Colorada. Calibración con OxCal y curva ShCal20.

En base a estos antecedentes, el presente trabajo realiza una caracterización bioantropológica de la población de Iglesia Colorada, investigando además la presencia de patrones de actividad de trabajo, entre los que se cuenta por ejemplo, el caso de la minería. De este modo podremos obtener una línea base para comparar con otros sitios y periodos, además de determinar el grado de impacto de la expansión Inca en relación a condiciones de vida y actividades laborales de la población local.

\section{MATERIAL Y MÉTODO}

Los restos humanos recuperados del sitio Iglesia Colorada se encuentran depositados en el Museo Regional de Atacama (sepultura 3 a 19) y en el Museo Nacional de Historia Natural (sepultura 1 y 2). Se analizaron los restos esqueletales provenientes de las sepulturas 1, 2, 3, 4, 5, 6, 7, 8, 9, 10, 11, 12, 16, $17 \mathrm{y}$ 19, del sector El Damasco y las dos sepulturas provenientes del Fundo Bauzá. Debido a la falta de análisis de la colección del sector el Damasco, fue necesario estimar el número mínimo de individuos. Una vez individualizados, se estimó la edad, el sexo, estatus dental, presencia de patologías e indicadores de estrés.

Para la estimación de edad se usó un conjunto de métodos de acuerdo a la viabilidad de su aplicación. En los cráneos, la edad se estimó mediante la observación del cierre de suturas maxilares (Mann et al. 1991) y de suturas craneanas individuales (Szilvássy 1988). También se consideró la fusión tardía de epífisis, como los anillos de los cuerpos vertebrales (Buikstra y Ubelaker 1994; Schaefer, et al 2009). En individuos completos se analizaron los cambios en la superficie auricular del coxal (Buckberry y Chamberlain 2002), los cambios en la superficie de la sínfisis púbica (Brooks y Suchey 1990) y los cambios en el extremo esternal de la cuarta costilla (Isçan et al 1984a;1984b: en Alsup 2007). La edad de los subadultos se estimó 
a partir del grado de fusión de los centros de osificación secundarios, la longitud de las diáfisis (Schaefer et al. 2009; Scheuer y Black 2004) y el desarrollo dental (Massler et. al. 1941; Rai et al. 2014).

Las edades fueron agrupadas en los siguientes rangos: infante (0-2 años), niñez temprana (3-5 años), niñez tardía (6-10 años), juvenil I (11-14 años), juvenil II (15-20 años), adulto joven (21-30 años), adulto medio (31-40 años), adulto maduro (41-50 años) y adulto senil ( +50 años). En los casos donde el estado de conservación no permite realizar estimaciones con precisión, se definió como adulto ( $>18$ años) y subadulto $(<18$ años $)$.

Para la estimación de sexo en individuos sobre la categoría Juvenil II, se analizaron los rasgos dimórficos del cráneo (Acsadi y Nemeskeri 1970; Ubelaker y Buikstra 1994), hueso púbico (Phenice 1969), forma de la escotadura ciática (Walker et al. 1988), surco preauricular y diámetro de la cabeza humeral (Bass 2005). La estimación de estatura se realizó a partir del método de estimación de Del Angel y Cisneros (2004), basado en Genovés (1967) para población amerindia (Del Angel y Cisneros, 2004). La toma de mediciones de huesos largos se realizó de acuerdo a las definiciones de Buikstra y Ubelaker (1994: 74-84).

Para abordar el estado dental se inventarió los dientes presentes siguiendo el protocolo FDI (KeiserNielsen 1971). Se registró el estado dental (diente y hueso perdido, perdido en post-mortem, perdido intravivam, diente presente y germen de diente), grado de desgaste (según la escala de Molnar 1971), presencia y grado de cálculo dental (Brothwell, 1981; en Hillson, 1996), cambio periodontal, presencia y tipo de caries (lesión en esmalte, en dentina, cavidad pulpar expuesta y diente o corona destruida). Los parámetros se definieron de acuerdo a Hillson (1996), Cucina (2011) Pinhasi y Mays (2007), Jacobi y Meyer (2007) y Molnar (1971).

Se registró la presencia de patologías que afectan a la morfología y textura del hueso como: trastornos metabólicos, daño articular y entesopatías. El estrés o disrupción fisiológica es una respuesta al empobrecimiento de las condiciones ambientales, causadas tanto por factores naturales como culturales (Cucina, 2011; Miquel-Feucht et al., 1999; Walker et al., 2009). Este se expresa en algunas alteraciones del tejido óseo y dental como es el caso de los síndromes cribosos: hiperostosis porótica, criba orbitaria y criba femoral e hipoplasia del esmalte (Roberts y Cox, 2003; Schultz et al., 1998; Stuart-Macadam, 1985). La criba orbitaria (CO) se ha asociado a la anemia y es una de las condiciones metabólicas más comunes del pasado (Gómez y Eggers, 2014; Miquel-Feucht et al., 1999; Stuart Macadam y Kent, 1992; Roberts y Manchester, 1996). Se observa como poros en el techo de la órbita, e incluso inflamación. El registro se realizó a partir de la clasificación de Schultz (1988) donde: leve= no supera 1/3 del techo de la órbita con orificios aislados; moderado= más de $1 / 3$ del techo de la órbita con porosidad, unión de los defectos y poca expresión de formación ósea nueva; severa $=$ crecimiento trabecular afuera de la diploe orbitaria. La hiperostosis porótica (HP) se caracteriza por lesiones craneales como el resultado de una hiperplasia de la médula interna causando un debilitamiento de la tabla externa. Se registró de acuerdo a Stuart-Macadam (1989) y Suby (2014): leve= con foramina fina dispersa; moderado= foramina aislada grande y pequeña con algunos de los forámenes fusionados para formar trabéculas; severo= crecimiento en la estructura trabecular del contorno normal de la tabla externa del hueso.

La criba femoral, es el síndrome criboso menos estudiado, de etiología poco clara, aunque también se la asocia a la anemia (Miquel-Feucht et al., 1999). Se la reconoce como un parche de porosidad en el cuello del fémur hacia inferior. Se registró de acuerdo a los siguientes parámetros: leve= parche con microporosidad no supera los $10 \mathrm{~mm}$ en el segmento inferior del cuello del fémur; moderado= parche con macroporosidad que cubre una superficie de $10 \mathrm{~mm}$ o superior; severo= crecimiento trabecular e inflamación en el área afectada.

La hipoplasia del esmalte dental es un defecto macroscópico causado por una disrupción durante 
la formación de la superficie de la corona (amelogénesis) (Cucina, 2011). Se observa como depresiones en forma de banda (hipoplasia de esmalte lineal, LEH) o como pozos en la superficie dental (Ortner y Putschar 1981: pag. 258-263; Cucina, 2011; Schultz et al. 1998). Las causas comúnmente se atribuyen a tensiones fisiológicas, como la malnutrición, enfermedades infecciosas, traumas psicológicos o físicos y otros trastornos metabólicos (Goodman y Rose 1991; Goodman y Goodman A H 1988). Se registró de manera macroscópica la presencia en cada diente y si se manifiesta como líneas (simples o múltiples) y/o pozos.

El análisis de actividades se centró en la presencia de lesiones articulares asociadas a procesos no degenerativos (Britges 1992; Jurmain y Kilgore 1995; Resnick y Niwayama 1981, Burt et al. 2013, Calce et al. 2018, Cheverko y Bartelink 2017). Se distinguió entre la osteofitosis vertebral (OV, o Spondylosis deformans) y la osteoartritis (OA o artrosis) de las articulaciones sinoviales, incluyendo en esto último las facetas apofisarias de la parte posterior de la columna vertebral (Bridges 1992; Burt et al. 2013; Jurmain y Kilgore 1995). El grado del daño articular se definió a la a escala: leve= signos patológicos incipientes; labiamiento suave y micro-porosidad; moderado= presencia de erosión, porosidad y osteofitosis en menos del 50\% de la superficie articular sin alterar la morfología y función del hueso afectado; o severo= alteración en la forma del hueso, cubriendo grandes áreas y/o alterando la función del hueso afectado, presencia de eburnación. Para la OV se suma la presencia de anquilosis (Lovell 1994; Aufderheide 1998; Ortner 2003). También se evaluó la presencia de indicadores de estrés músculo esqueletal mediante la revisión de entesopatías. En este caso se evaluó su presencia y tipo de alteración considerando procesos osteofíticos u osteolíticos en áreas de entesis (Mariotti et al. 2004; Villotte et al., 2016; Villotte et al., 2010). Finalmente se sumó al análisis el reconocimiento de alteraciones en la morfología del hueso donde se discute si estas son el resultado de actividades (Molleson, 2007).

\section{RESULTADOS}

Los restos provenientes del sector el Damasco representan un número mínimo de 27 individuos (cuadro 1). A éstos se sumaron los restos humanos de los cuatro individuos recuperados del Fundo Bauzá. La muestra por tanto asciende a 31 individuos. Cabe señalar que los restos humanos recuperados en el Potrero El Damasco se encuentran en malas condiciones de preservación, mediana o pobremente representados, muy fragmentados, con alta pérdida de colágeno y erosión en sus superficies. Los restos humanos recuperados en el Fundo Bauzá, en cambio, presentan condiciones totalmente distintas. El sedimento rico en calcita del sector permitió que los restos humanos de infantes se momificaran y se preservaran sus fardos completos, incluyendo textiles y cestería. El individuo adulto recuperado en dicho sector se encuentra esqueletizado completo y en buen estado. Del total de la colección, el 64,5\% de los individuos se encuentran incompletos ( $50 \%$ a $1 \%$ del esqueleto), sólo un $9,7 \%$ semicompletos y $25,8 \%$ se encuentran completos.

\section{PERFIL DE LA POBLACIÓN}

Los 31 individuos analizados (cuadro 2) corresponden a un nonato, siete lactantes, un niño de 4 años ( \pm 12 meses), un niño de 6 años ( \pm 12 meses), un juvenil de 12 años ( \pm 30 meses), cinco juveniles de entre 14 y 20 años, y 14 adultos (de entre 20 y 50+ años). Solo pudo estimarse el sexo en nueve de los 17 individuos mayores de 15 años. De éstos, los individuos femeninos comprenden un $24 \%$ y los individuos masculinos un 13,8\% (figura 2). La mayoría de las mujeres falleció entre los 15 y los 30 años, mientras que los hombres fallecieron entre los 30 y los 40 años.

Solo se pudo estimar la estatura en el individuo 1 de la sepultura 10 (femenino de 18 a 20 años). Usando la fórmula de Genovés (1967) esta se calculó en 1,59 metros, lo cual es similar con lo observado por Ericksen (1978) para poblaciones prehispánicas del valle del Elqui asociadas a la cultura Diaguita. 


\section{CONDICIONES ORALES}

Se analizaron los dientes permanentes de 16 individuos con edades entre la niñez tardía y la adultez senil. Se calculó que en total los 16 individuos deberían tener 512 dientes (asumiendo 32 dientes por cada uno). De este total hipotético, sólo fueron identificados 175 dientes $(34,2 \%)$, se estableció la pérdida en vida de 57 dientes (11,1\%), 30 dientes en desarrollo (5,9\%) y los restantes 250 dientes $(48,8 \%)$ están ausentes. Los dientes deciduos solo se usaron para estimar edad, por lo tanto no forman parte de este análisis.

Los maxilares y mandíbulas en su mayoría están incompletos, por lo tanto sólo se pudo registrar 79 alveolos, de estos $21,5 \%$ no presenta retracción. La mayor frecuencia se concentra en la retracción baja $59,5 \%$ afectando las crestas interdentales. Un 16,5\% presenta retracción moderada y sólo un 2,5 (dos casos) presenta retracción alta.

La pérdida dental en vida (intra vivam) se observó en sólo tres individuos, aunque no se puede descartar que haya afectado a otros individuos cuyo hueso alveolar está ausente. Esta pérdida dental se observó en dos adultos mayores de 45 años, enterrados en sepulturas con ofrendas, pero destaca el caso de la segunda cabeza de la sepultura 19, que registró una pérdida de 11 dientes, condición atípica, considerando que se trata de una mujer joven de 25 a 35 años.

El desgaste dental presentó un promedio bajo de 2,51 con 1,4 de la desviación estandar (DE), para la escala de Molnar (1971) con grados del 1 al 8 (Figura 2). El único caso que presenta un desgaste acentuado es el individuo CNCR LA-2016.02.04 con una edad mínima de 45 años y un desgaste entre 6 y 8 . El alto desgaste observado en los incisivos de este individuo se explica por la pérdida de los premolares y molares. En el resto de los individuos, los grados van de 1 a 3, y los dientes anteriores tienden a presentar grados más leves. Mediante la prueba de Tau-c de kendall para muestras pequeñas, se observó una correlación moderada entre el grado de desgaste y la edad (cuadro 3), posiblemente debido al tamaño reducido de la muestra ${ }^{1}$.

\footnotetext{
1/ Los datos no se distribuyen de forma normal.
}

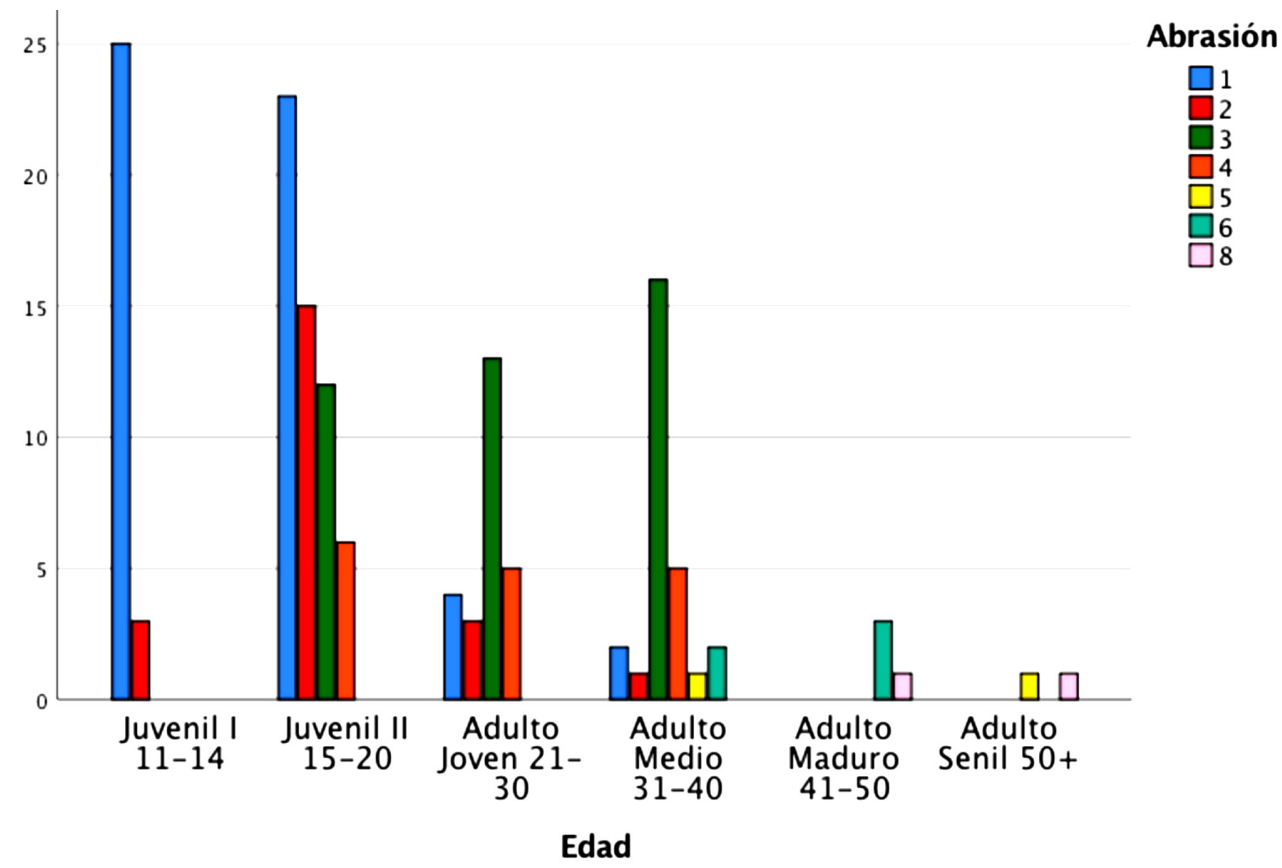

Figura 2: Frecuencias de piezas dentales ordenadas de acuerdo al grado de desgaste dental y grupo etario. 
La presencia de cálculo dental en la muestra analizada es baja. Un 19,6\% de los dientes observados (175 dientes) mostró depósitos pequeños, un 1,2\% presentó depósitos medianos y no se observó en grados severos. Solo fueron registrados abscesos en el individuo CNCR LA-2016.02.04, asociados a desgaste severo y pérdida dental.

En los 175 dientes disponibles, se registraron siete caries en cuatro individuos: una cervical (grado 1; pequeña) en el individuo 1 de la sepultura 1 , dos caries oclusales en el individuo 1 de la sepultura 8 , tres caries oclusales pequeñas en el individuo 1 de la sepultura 10, y una pequeña oclusal en el individuo 2 de la sepultura 10. Las caries registradas representan solo un 3,9\% de los dientes presentes y se observaron únicamente en primeros y segundos molares.

\section{INDICADORES DE ESTRÉS FISIOLÓGICO}

En los individuos de Iglesia Colorada la hiperostosis porótica (HP) fue observable sólo en 13 de los 31 individuos de la muestra, a causa del mal estado de conservación de los cráneos. Dentro de los individuos analizados se registró HP recuperada en el 57,1\% de los individuos (7). No se registró en infantes, sólo entre juveniles y adultos jóvenes (entre 12 y 30 años).

La presencia de criba orbitaria sólo pudo evaluarse en cuatro individuos de la muestra que conservan los techos de las órbitas. En ninguno fue registrada. La criba femoral sólo pudo ser observada en seis individuos, registrándose su presencia en grado leve en el individuo 1 de la sepultura $1 \mathrm{y}$ en el individuo 2 de la sepultura 10. La hipoplasia del esmalte pudo ser registrada en 14 individuos, de los cuales 13 la presentan (el único que se excluye es el individuo 1 de la sepultura 10). 7 individuos presentan sólo una línea (grado 1), siete individuos presentan más de una línea (grado 2), y no se observó hipoplasia en grado severo. De los 175 dientes analizados se registró hipoplasia en el 15,4\% de estos.

\section{ARTROPATÍAS Y ALTERACIONES EN LA MORFOLOGÍA DEL HUESO.}

Aunque la mayoría de los individuos analizados presentan erosión en el tejido óseo, pérdida de tejido trabecular y de las epífisis, fue posible observar la presencia de artropatías en los esqueletos más completos, principalmente de las sepulturas 1, 5, 10 y 11. En el caso de las entesopatías, estas sólo se observaron en las clavículas de dos individuos, y son coincidentes. El detalle de las observaciones se describe a continuación.

Individuo 1, sepultura 1 (I1-S1), masculino de 40 a 50 años de edad. Es uno de los esqueletos mejor preservados. Conserva la mayoría de sus vértebras y superficies articulares. Presenta daño articular general leve en comparación con los otros individuos analizados. Destaca la presencia de nódulos de Schmorl en la primera, segunda y cuarta vértebra lumbar (Figura 3), leve osteoartritis en las articulaciones cigapofisarias de la cuarta y quinta lumbar y osteoartritis moderada en el cóndilo femoral derecho, con alteración de la superficie articular, macroporosidad y hueso reactivo (Figura 3).

Individuo 1, sepultura 5 (I1-S5), posiblemente masculino de 30 años o más. Su columna vertebral está representada sólo por la vértebra atlas, dos vértebras torácicas fragmentadas y una vértebra lumbar. En dos de las cuatro vértebras presentes se observaron artropatías severas. El atlas presenta labiamiento y deformación de la superficie articular en el cóndilo izquierdo y división en la superficie condilar derecha (Figura 4). La vértebra lumbar presenta osteofitos y porosidad severa (Figura 4).

Individuo 1, sepultura 10 (I1-S10), femenino de entre 18 y 20 años. Corresponde a uno de los esqueletos más completos de la colección. Presenta osteoartritis en las facetas esternales de ambas clavículas 

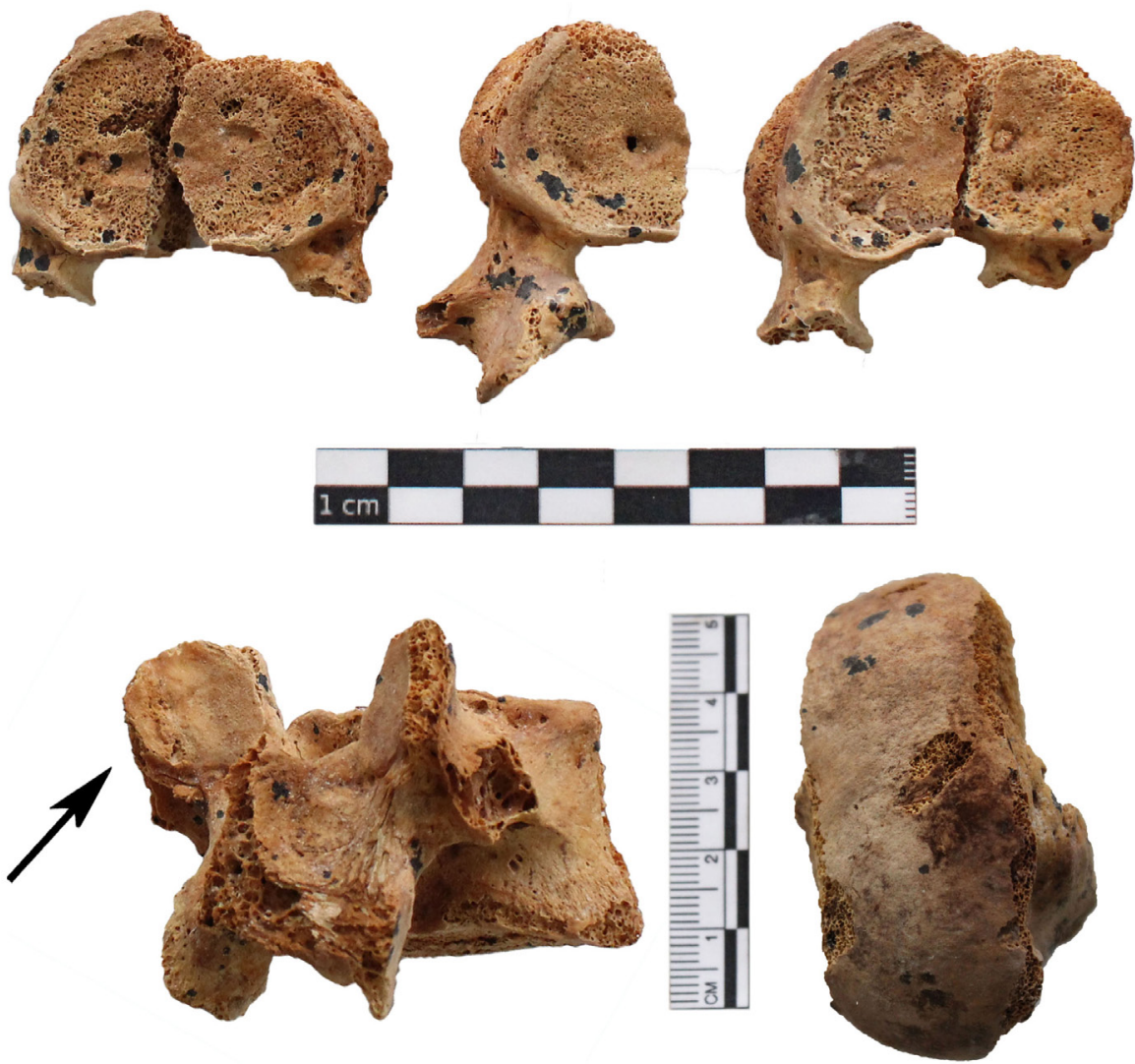

Figura 3: Arriba, nódulos de Schmorl en L1, L2 y L4, individuo de la sepultura 1. Abajo a la izquierda, osteoartritis en articulación cigapofisaria. Abajo a la derecha, osteoartritis en cóndilo femoral derecho.
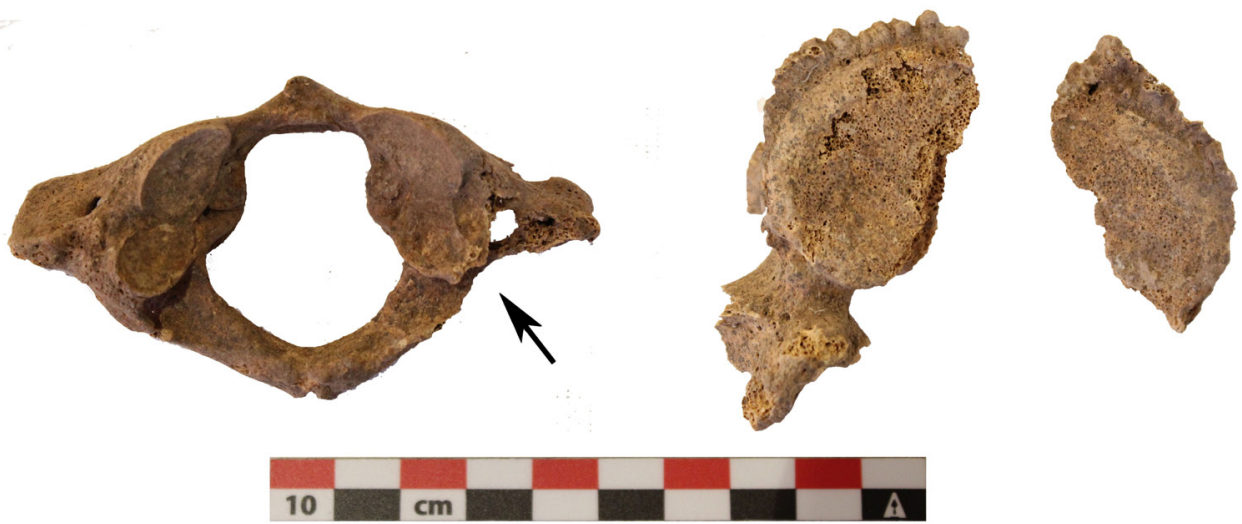

Figura 4: Individuo 1 de la sepultura 5. A la izquierda, atlas con osteoartritis moderada en cóndilo y articulación atlantooccipital dividida. A la derecha, osteofitosis en vértebra lumbar. 
(Figura 5-A) y una marcada impresión costo-clavicular (sindesmosis). La columna muestra daño en todo el segmento torácico y lumbar. En las vértebras torácicas se observó labiamiento leve en los bordes anteriores de los cuerpos y osteoartritis unilateral (con porosidad y osteofitos) en las articulaciones cigapofisarias derechas de T2, T3 y T4 (Figura 5-B), las cuales sugieren posible escoliosis. Los bordes anteriores de los cuerpos de las vértebras lumbares también muestran labiamiento leve (Figura 5-C). Ambas patelas presentan escotaduras en el borde meso-superior (Figura 5-D). En ambas falanges distales del Hallux (primer dedo del pie) se observó una leve artropatía (Figura 5-E), que podría corresponder a "Hallux valgus interfalangeal". También se registró una proyección en la faceta flexora lateral de la tróclea del astrágalo y un labiamiento leve en los huesos del tarso.

Individuo 1, sepultura 11 (I1-S11), posible femenino, de 18 a 25 años. En la cara mesial de la patela derecha se observó una osteocondritis disecante de $7 \mathrm{~mm}$ de diámetro con bordes limpios (Figura 6).

\section{A)}
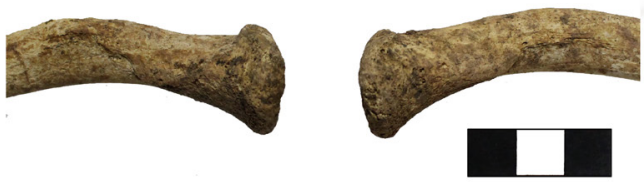

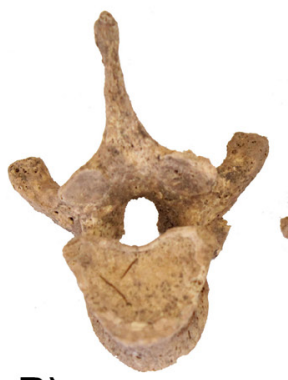

B)
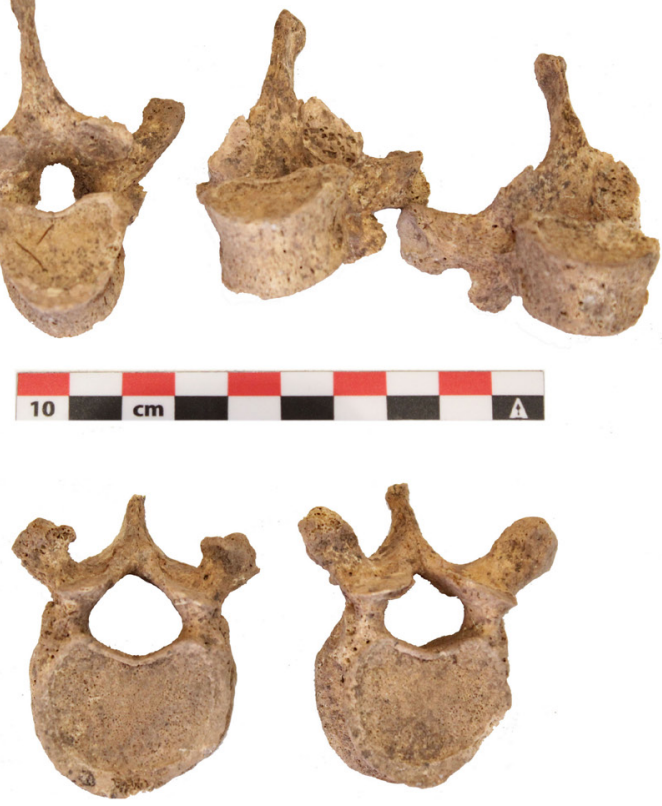

D)
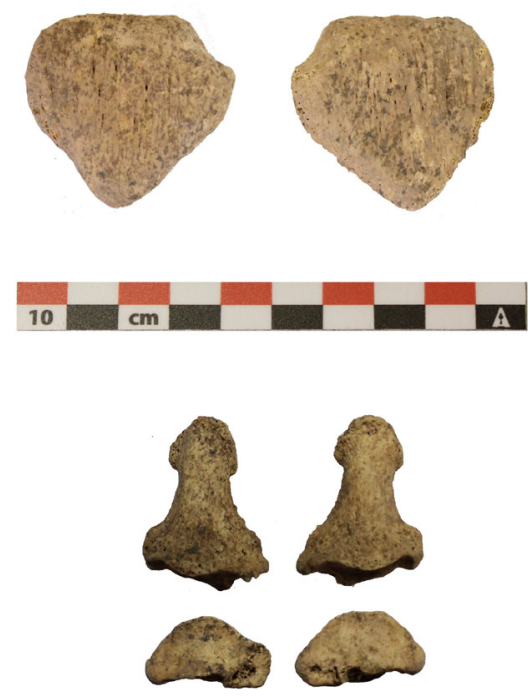

E)

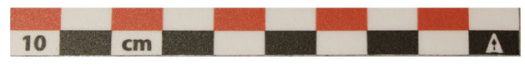

Figura 5: Individuo 1 de la sepultura 10. A) Osteoartritis en las facetas esternales de ambas clavículas. B) Vértebras torácicas con macroporosidad y osteofitosis en los contornos de las articulaciones cigapofisarias. C) Leve labiamiento en los bordes anteriores de los cuerpos de las vértebras lumbares. D) Escotaduras en ambas patelas. E) Artropatías en falanges distales de Hallux derecho e izquierdo. 
Individuo 2, sepultura 11 (I2-S11), posiblemente masculino de entre 35 y 40 años. Su esqueleto se encuentra fragmentado e incompleto, pero se pudo observar erosión en el cuerpo vertebral de la cuarta cervical, osteofitosis severa en C3, C4 y C5 (Figura 7-A) y osteofitosis moderada en la tercera y cuarta lumbar. También presenta osteoartritis erosiva en las facetas esternales de ambas clavículas y entesopatía en la impresión costo-clavicular (Figura 7-B).

Las vértebras cervicales del individuo 3 de la sepultura 19 (I3-S19) se encontraron incompletas y erosionadas, pero se pudo observar una pequeña irregularidad (hundimiento) en la superficie articular del cóndilo izquierdo del axis (Figura 8), la cual podría corresponder a un defecto de desarrollo o a una osteocondritis disecante.

\section{DISCUSIÓN}

A fin de ofrecer un perfil de la población, con una muestra reducida, a continuación se discuten las evidencias en relación a la bibliografía pertinente y en comparación con antecedentes de otras poblaciones de las culturas Copiapó y Diaguita.

\section{PERFIL DEMOGRÁFICO}

De los 31 individuos analizados, $29 \%$ son lactantes y 45,2\% son adultos. La estructura demográfica del cementerio es esperable para poblaciones preindustriales donde las principales causas de muerte son infecciones gastrointestinales y respiratorias (Lewis 2007; Volk y Atkinson 2013). También cabe ponderar el proceso del destete y sus consecuencias a nivel de dieta y sistema inmune como un factor importante en la mortalidad infantil (Katzenberg et al. 1996). Los nueve individuos con sexo estimado incluyen 7 individuos femeninos (24\%) y 3 individuos masculinos $(13,8 \%)$. El antecedente demográfico más cercano, aunque
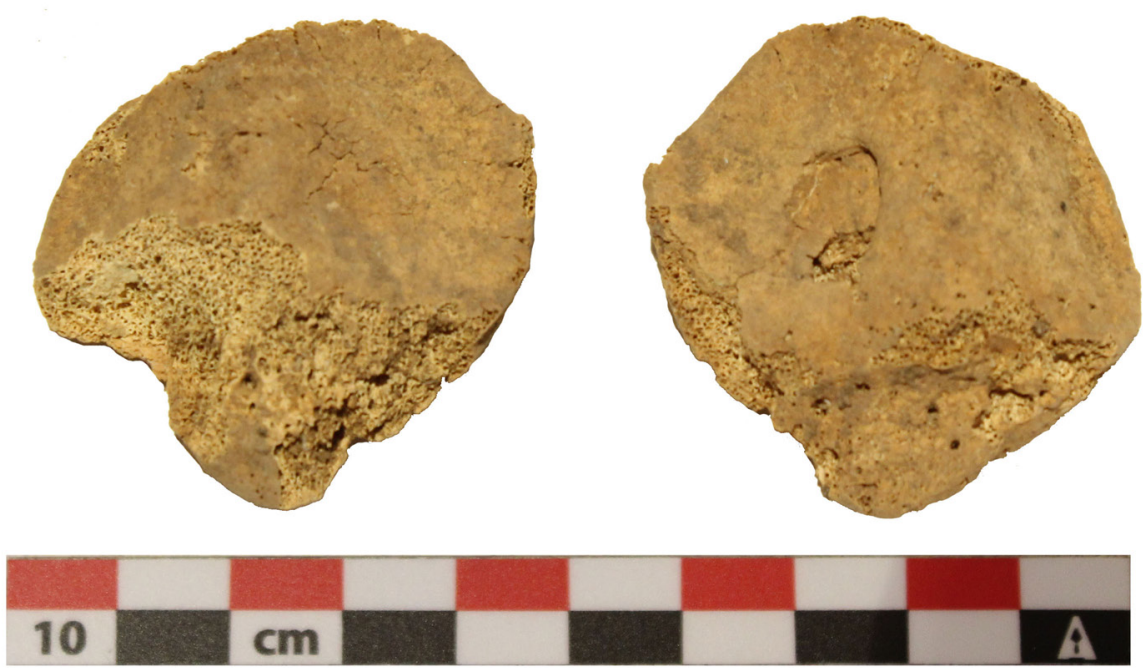

Figura 6: Individuo 1 de la sepultura 11. Osteocondritis disecante en patela derecha. 


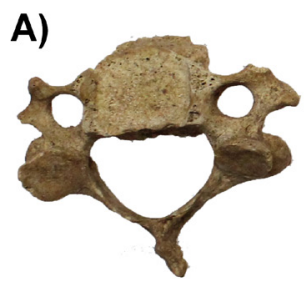

B)
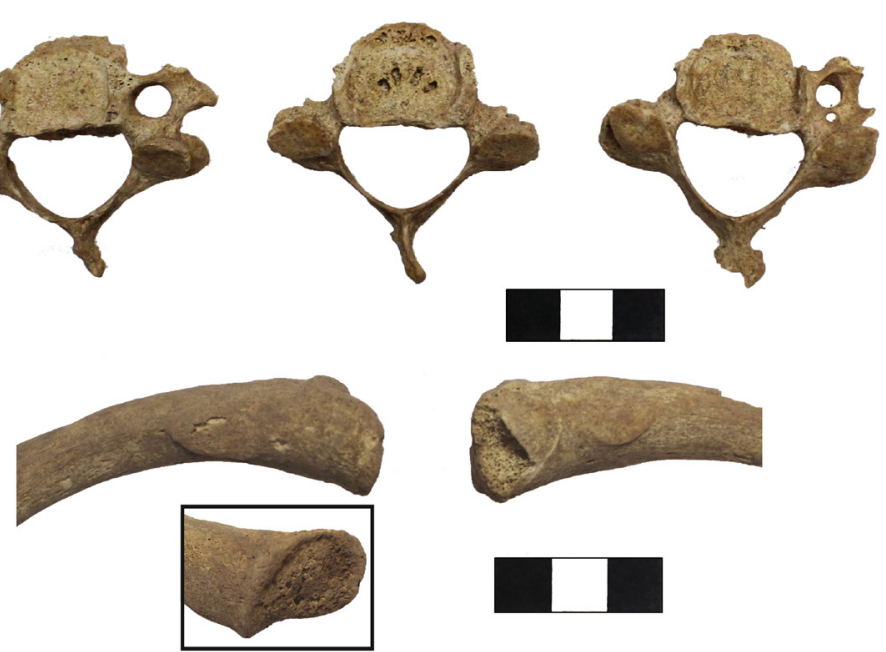

Figura 7: Individuo 2 de la sepultura 11. A) Osteofitosis en C3, C4 y C5. B) Osteoartritis erosiva en las facetas esternales de ambas clavículas y entesopatía en la impresión costo clavicular

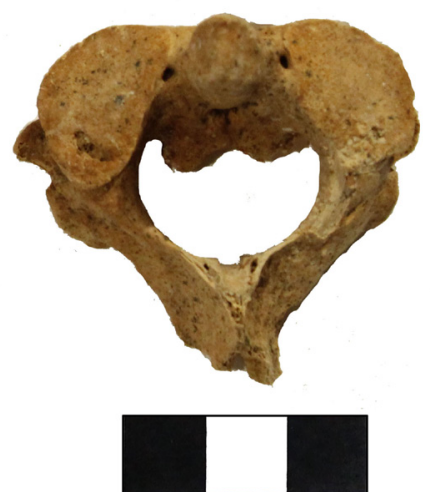

Figura 8: Individuo 3 de la sepultura 19. Posible osteocondritis disecante en axis.

más temprano, es el sitio cementerio La Puerta localizado en el curso medio del valle de Copiapó, en donde se analizaron 66 individuos procedentes de 48 túmulos funerarios correspondientes al Periodo Medio y asociados a la cultura Ánimas (Niemeyer 1998). Para La Puerta se estableció un 27.7\% de lactantes, 19.9\% de infantes y juveniles y un $46.1 \%$ de adultos, muy similar a lo informado en el sitio Iglesia Colorada. Las diferencias en las edades de muerte entre hombres (30-40 años) y mujeres (15-30 años) podrían explicarse porque una de las principales causas de muerte en mujeres jóvenes se asocia a la gestación y el parto (Sayer y Dickinson 2013), sin embargo esta diferencia también puede estar influida por el entierro de cabezas aisladas, en donde 3 de los 4 casos corresponden a individuos femeninos jóvenes (Garrido y Morales 2019).

\section{CONDICIONES ORALES}

Dentro de la muestra destaca el caso del segundo cráneo de la sepultura 19 (mujer joven de 25 a 35 años), quien registra una pérdida de 11 dientes. La pérdida dental a temprana edad en mujeres puede ser una consecuencia de la gingivitis gestacional, sin embargo estas pérdidas no superan los dos dientes por dentadura (Fields et al. 2009). Por esta razón se estima que la pérdida dental masiva pueda atribuirse a una 
situación de estrés particular, por causas internas o externas. La ausencia de otros indicadores y de la mayor parte del esqueleto no permiten profundizar en el caso.

En general, el desgaste dental observado fue bajo, excepto en el individuo CNCR LA-2016.02.04. El desgaste comienza alrededor de los 18 años, pero no aumenta hasta al menos los 40 años y luego, a partir del rango "adulto maduro", se reconoce un aumento considerable asociado a la pérdida de piezas dentales. El desgaste con predominio de grados moderados es típico de grupos con un desarrollo agro-ganadero y dietas poco abrasivas (Fields et al. 2009). Este patrón es similar a lo observado en el individuo 29 del sitio Caserones 61, femenino de alrededor de 23 años, con cronología adscrita al PT y fechado entre 1410-1470 d.C. cal. ${ }^{2}$ (Díaz y Pacheco 2017; Rodríguez y Pizarro 2015). En poblaciones del norte semiárido adscritos a la Cultura Diaguita (períodos PIT y PT, en los sitios Peñuelas 21 y 24, Planta Pisco Control, PeñuelasCONAF, El Olivar e Illapel), se han descrito grados de desgaste moderado, con diferencias según edad (Araya-Rosado y Vernacchio-Wilson 2006). Andrade (2012), por su parte, distinguió un desgaste moderado en poblaciones Diaguita de la costa (PIT, sitio Los Coiles 136) y leve en las del interior (sitios Estadio de Illapel, Loma El Arenal, Valle Hermoso y MLP PTF 28).

El bajo cálculo dental (19,6\% leve, 1,2\% moderado y sin grados severos), es similar a lo observado por Andrade (2012) en la muestra diaguita del interior (4,87\%), con una marcada diferencia con respecto a la costa (40,25\%). El cálculo dental suele estar asociado a dietas ricas en proteína animal, como las de los cazadores recolectores, principalmente costeros (Cucina, 2011). Es posible que el consumo de proteína animal terrestre no genere tanto depósito de calcio.

La enfermedad periodontal observada se registró principalmente de modo leve $(59,5 \%)$, son un $16,5 \%$ moderada y $2,5 \%$ alta. Se observó como retracciones regulares con obliteración inicial de las crestas alveolares y más asociado a la atrición que a procesos infecciosos (Pinhasi y Mays 2007). Solo se registraron abscesos en un individuo, pero es posible que este indicador esté subestimado debido a la preservación deficiente del material óseo.

La baja prevalencia de caries (3,9\% de la muestra) en Iglesia Colorada es similar a lo que se registró en el individuo 29 del sitio Caserones 61 (PT), con solo una lesión cariosa (Rodríguez y Pizarro 2015, Pacheco 2016). En las poblaciones diaguitas del PIT y PT de la Región de Coquimbo la prevalencia de caries es considerablemente más alta. En Peñuelas 21 y 24 los dientes afectados alcanzan un 63\% de la muestra (Araya-Rosado y Vernacchio-Wilson 2006), y en Punta de Piedra se mencionan extensas patologías dentarias, donde un solo individuo puede llegar a presentar hasta cinco caries, incluyendo daño en la cavidad pulpar (Ericksen, 1978). Tanto Araya-Rosado y Vernacchio-Wilson (2006) como Ericksen (1978) asocian la alta prevalencia de caries al consumo de plantas cultivadas y al desgaste dental. En el estudio comparativo de Andrade (2012) las caries presentaron porcentajes de 10,84\% en los Diaguitas de la costa y $16,09 \%$ en el interior.

En síntesis y pese a la incompletitud de la muestra dental, fue posible observar que los individuos de Iglesia Colorada presentan dentaduras con poco desgaste, producto de una dieta blanda, posiblemente agrícola, aunque curiosamente baja en caries. De acuerdo a Lukacs y Largaespada (2006), la falta de caries dental puede ser un indicador de buena salud, y no puede descartarse la presencia de fluoruros en el agua, los cuales también pueden evitar la proliferación de caries bucales (Beckett y Lovell 1994; Cucina 2011; Hillson 1996; Irish et al. 2015). Los depósitos de cálculo dental y la enfermedad periodontal son leves y similares a los observados en poblaciones Diaguita del interior (Andrade 2012). Hasta el momento no se han realizado estudios de dieta de la población de Iglesia Colorada, pero a partir de lo observado en las ofrendas y el contexto histórico y geográfico, se puede inferir el consumo de alimentos cultivados donde

2/ Fechas calibradas con la curva SHCal 13 (Hogg et al. 2013), con 2 sigmas en OxCal 4.3 de acuerdo a Bronk Ramsey (2009) (Díaz y Pacheco 2017). 
destacan el maíz y el chañar, ambos ricos en azúcar. No existe claridad sobre cuáles fueron las formas de preparación de estos alimentos, pero al menos en el caso del maíz es posible que se haya privilegiado su consumo en forma de chicha (bebida alcohólica fermentada).

\section{INDICADORES DE ESTRÉS FISIOLÓGICO}

La causa de la HP no es del todo clara, pero comúnmente se ha asociado a la anemia por falta de hierro (Stuart-Macadam 1992; Roberts y Manchester 2010). Sin embargo, la deficiencia de hierro puede ser más una consecuencia que la causa (Rothschild 2012). Esta puede obedecer a mecanismos más complejos, con énfasis en las deficiencias nutricionales y/o condiciones infecciosas que se potenciarían con el estrés nutricional (El-Najjar et al. 1976; Lallo et al. 1977; Stuart-Macadam et al. 1989). En Iglesia Colorada se registró hiperostosis porótica (HP) en 7 de los 13 individuos observables $(57,1 \%)$. En ninguno de los casos es severo, ni asociado a infantes. Este indicador es frecuente en poblaciones preindrustriales (Ortner 2003), por lo tanto su presencia en grados leves y moderados no indica un estrés fisiológico considerable.

No se registró criba orbitaria en los cuatro individuos observables. La criba femoral, por su parte, se registró en dos de los seis individuos observables. Esta es considerada como parte del síndrome criboso, pues se estima que comparte una etiología similar a la HP y la criba orbitaria (Miquel-Feucht et al. 1999), aunque se ha indicado su posible asociación con la falta de magnesio (Rude 1998).

El análisis de hipoplasia del esmalte se centró en la presencia de bandas o surcos lineales, (hipoplasia del esmalte lineal o LEH en inglés), considerando que esta tiene mayor relación con disrupciones metabólicas sistémicas. En la muestra analizada, fue registrada en 13 de 14 individuos analizables, sin presentar casos severos. La edad en la que se forman los defectos hipoplásicos en los dientes permanentes anteriores (incisivos y caninos) corresponde a los primeros 2 años de vida, momento asociado con la etapa del destete (Katzenberg et al., 1996; Wright y Schwarcz, 1998). En este momento los infantes son susceptibles a sufrir estrés nutricional y vulnerabilidad ante nuevos patógenos. Por esta razón las hipoplasias menores e hipomineralizaciones aparecen con sorprendente frecuencia en los dientes de niños aparentemente sanos, y están presentes en un 43-64\% de los individuos de las poblaciones modernas (Cucina, 2011; A. H. Goodman y Armelagos, 1989; Katzenberg et al. 1996; Pinhasi y Mays, 2007). Por lo tanto, la presencia de indicadores medios y bajos de líneas de hipoplasia en Iglesia Colorada, no nos hablan necesariamente de una población afectada por el estrés fisiológico. Incluso, siguiendo la paradoja de Wood (1992), la presencia de grados moderados de hipoplasia lineal nos permite inferir que se trata de una población resistente a los procesos críticos del desarrollo (Pinhasi y Mays, 2007).

\section{ALTERACIONES EN LA COLUMNA VERTEBRAL}

En las articulaciones observadas destaca la presencia de osteofitosis a nivel cervical y lumbar, con nódulos de Schmorl (Figura 9). Estas evidencias configuran un patrón consistente y relacionado con las áreas de máxima curvatura de la columna (regiones cervical y lumbar) (Bridges 1992; Burt et al. 2013). Se ha establecido que las mayores frecuencias de OV se concentran en la región cervical (entre C5 y C6) y la región lumbar (entre L2-L4). En el segmento torácico esto es menos claro y varía entre T7 y T11, y que la región entre C7 y T3 siempre es la menos afectada por osteofitosis (Bridges 1992, Knüsel et al. 1997). Cabe mencionar al respecto, que los individuos I1-S5 e I2-S11, no siguen el patrón a nivel cervical (pues tienen afectadas las primeras cinco vértebras) pero sí hay consistencia a nivel lumbar. El I2-S10, además, es discrepante pues presenta OV leve y OA en las articulaciones cigapofisarias torácicas y lumbares del lado derecho. Dicha asimetría rara vez se ha examinado en detalle, pero es común en las vértebras, pues incluso puede estar relacionada con la posición de la aorta (ubicada en el lado izquierdo de la columna en ese nivel) (Bridges 1992). 

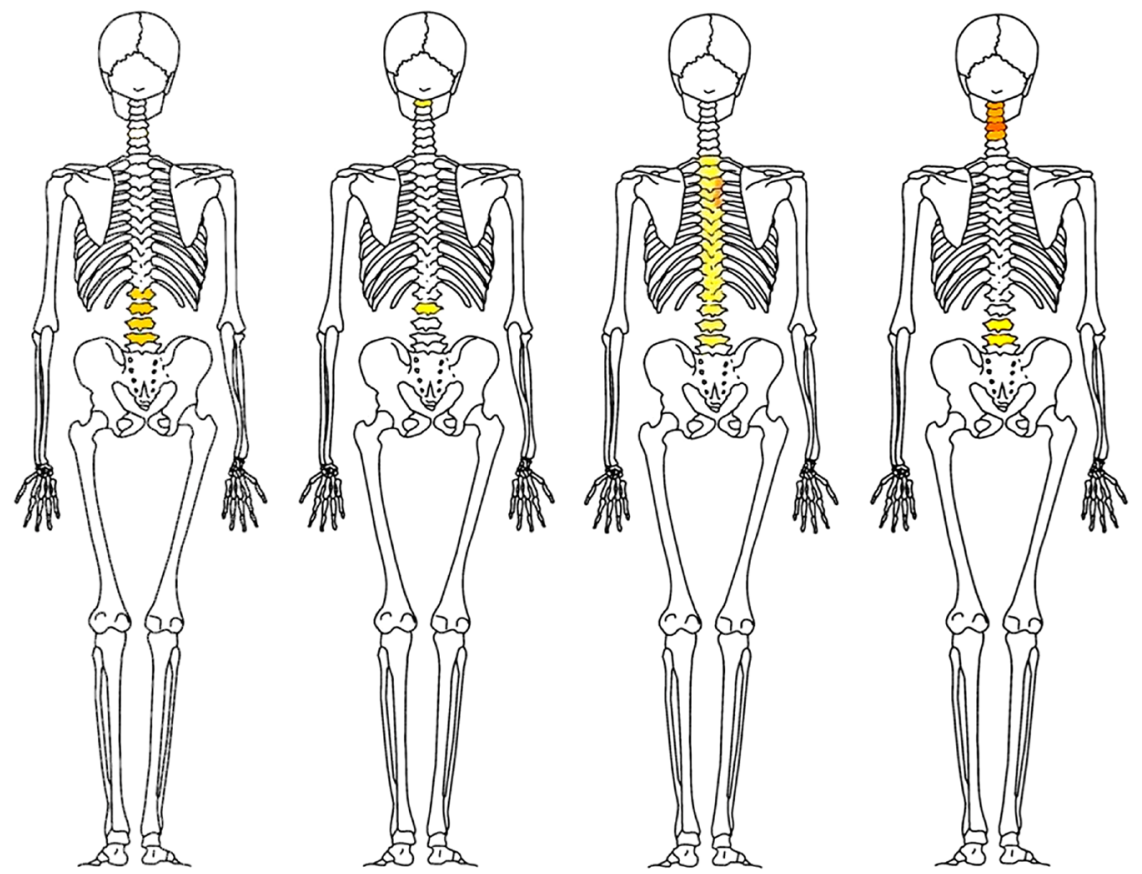

Figura 9: Localización de artropatías degenerativas en la columna vertebral. De izquierda a derecha, individuo 1 de la sepultura 1 (40-50 años, masculino); individuo 1 de la sepultura 5 (30+ años, masculino); individuo 1 de la sepultura 10 (18-20 años); individuo 2 de la sepultura 11 (35-40 años, posible masculino).

La división de la articulación atlanto-occipital de individuo I1-S5, comúnmente se considera como un rasgo discreto (Finnegan 1978; Buikstra y Ubelaker 1994); sin embargo, esto podría asociarse también a un estrés por actividad y movimientos repetitivos, o a la carga de peso sobre la cabeza, similar a lo observado por Molleson (2007) en el cóndilo occipital de las tejedoras de Spitalfields. Esto último coincide con la OV observada en el cóndilo izquierdo.

\section{ALTERACIONES EN EL ESQUELETO APENDICULAR}

Estas fueron menos claras de observar que las de la columna vertebral. Destaca la OA severa en la articulación esterno-clavicular bilateral de los adultos jóvenes I1-S10 e I2-S11, la cual puede atribuirse a actividades de carga que causan presión entre la clavícula y la primera costilla (Molleson, 2007; Robb, 1994), o bien podría ser resultado de actividades de molienda. En ambos casos coincide la presencia de entesopatías en el ligamento costo-clavicular, lo que reafirma la tesis de una alteración causada por actividad (figura 10).

La OA en las falanges distales del hallux de I1-S-10 (femenino de entre 18- 20 años), podría remitir a hallux valgus interfalangeal (HVI), lesión comúnmente atribuída a una condición genética y al uso de determinado tipo de calzado (Mays 2005; Nix et al. 2010), pero también a modificaciones por causa ocupacional. Molleson (1994) asoció el cambio en el ángulo medial del hallux y la extensión de la primera articulación metatarsofalángica al uso de elementos de molienda. Tal actividad requiere una presión considerable de la piedra de moler apoyada en las rodillas (hiperflectadas) con ambos halluces enroscados para ejercer fuerza sobre las rodillas y caderas.

La molienda también explicaría la faceta lateral del astrágalo y la muesca superomedial en las patelas de este individuo (Buchet 1989; Huard y Montagne 1951; Molleson 1994; Ubelaker 1979). Si bien, 

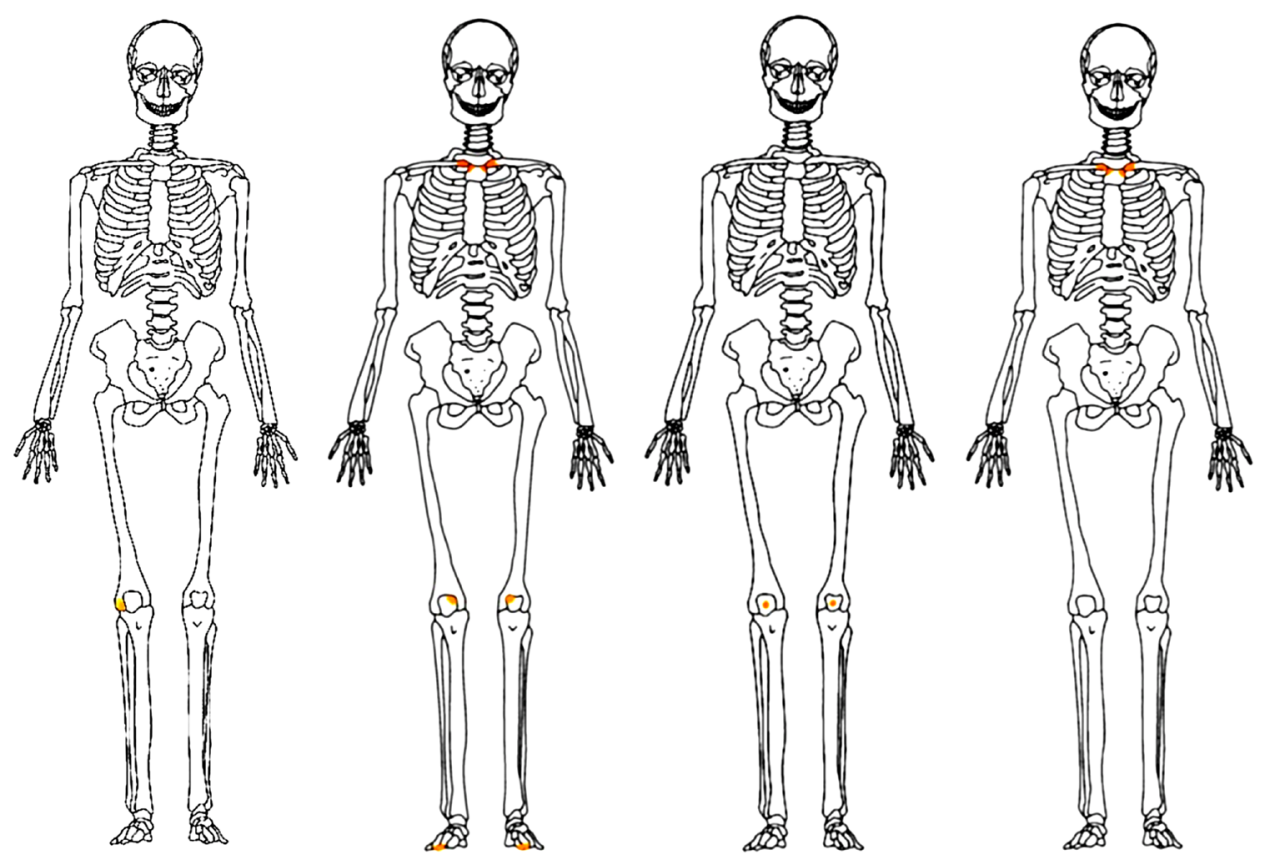

Figura 10: Localización de artropatías degenerativas apendiculares. De izquierda a derecha, individuo 1 de la sepultura 1 (40-50 años, masculino); individuo 1, sepultura 10 (18-20 años, femenino); individuo 1, sepultura 11 (18-25 años, femenino); individuo 2, sepultura 11 (35-40 años, posible masculino).

las muescas patelares se han interpretado como rasgos discretos (Finnegan 1978; Buikstra y Ubelaker, 1994), el conjunto de indicadores en I1-S10, sugieren ser interpretados como el resultado de la actividad de molienda.

El caso I1-S11 (femenino, 18-25 años) presenta una osteocondritis disecante (OCD). Pese a su etiología multifactorial, se la ha asociado a microfracturas causadas por eventos traumáticos repetitivos en individuos con alta demanda física, principalmente en individuos jóvenes, por lo tanto, puede ser un indicador de inicio temprano de labores con alta demanda física (Justus y Agnew 2010; Kocher et al. 2006; Kothari et al. 2009). Esta última interpretación se puede aplicar al sitio Iglesia Colorada, al menos en mujeres. Del mismo modo, nos puede hablar de una actividad física demandante. La propia geografía del lugar puede ser una causa de este tipo de lesiones. La OCD, se asocia a estilos de vida agrícolas con alta demanda (Cohen y Armelagos, 1984), y para este caso es posible proponer una relación con la actividad minera.

\section{INDICADORES DE ACTIVIDAD}

El estudio de patrones artropáticos como indicadores de estrés mecánico repetitivo causado por actividades culturalmente modeladas ha sido ampliamente discutido (Stewart 1958,1966; Angel 1966; Wells 1962; Bridges 1992; Jurmain y Kilgore 1995). Sin embargo, para este tipo de indicador se reconocen dos conflictos: en primer lugar la OA y OV resultan de una combinación de factores (Roberts y Manchester 2010) y, en segundo lugar, el sistema músculo-esqueletal responde de forma similar a diversos estímulos (Cheverko y Bartelink 2017). No obstante, es posible reconocer el énfasis de una población en el uso de determinados grupos articulares distintivos, así como la presencia de alteraciones óseas particulares. 
Hasta el momento, aparte del estrés mecánico, los factores de riesgo más ampliamente reconocidos son la edad y, en menor medida, el sexo, herencia, sobrepeso y traumas preexistentes (Roberts y Manchester 2010; Cheverko y Bartelink 2017).

Frecuentemente la OA y la OV se definen como "artropatías degenerativas". Se ha reconocido que la edad puede explicar hasta el 56\% de la variación en la gravedad de la OV (Calce et al., 2018; Felson, 2003). Tal relación ha servido como argumento para discutir la viabilidad de la osteoartritis como indicador de actividad (Knüsel et al. 1997). Sin embargo, esta relación no es lineal (Rogers y Dieppe, 2003). La OA-OV es poco frecuente en adultos menores de 40 años y extremadamente frecuente en aquellos mayores de 60 años (Calce et al., 2018; Felson, 2003). Una de las razones de esta irregularidad, es que el envejecimiento no es la causa directa, sino los cambios relacionados con la edad, aquellos que afectan tanto a la función articular como a los tejidos circundantes. Por lo tanto, no sólo se trataría de un mecanismo degenerativo, sino uno de reparación (Calce et al., 2018; Rothschild y Woods 2012). Esto es respaldado por estudios como el de Loudon (2016) y Weber (2003) donde se reconoce que la edad no ejerce un sesgo significativo en la prevalencia de artropatías cuando las colecciones se componen mayoritariamente de individuos menores de 45 años. Por lo tanto, un patrón en determinados segmentos de OA (y OV), cuya prevalencia sea alta en individuos jóvenes, podría ser un indicador confiable de actividad (Burt et al., 2013; Rojas-Sepúlveda y Dutour, 2014). Precisamente, un aspecto que destaca en los individuos de Iglesia Colorada es la presencia de lesiones a temprana edad, donde las artropatías y entesopatías se observan en individuos de entre 20 y 40 años.

En el caso del desarrollo de entesopatías el diagnóstico es similar, la edad es un factor de desarrollo en las alteración de las entesis. De acuerdo a Mariotti et al. (2004), las entesopatías osteofíticas son más frecuentes en adultos maduros que en jóvenes y viceversa. Por lo tanto la presencia de entesis osteofítica en jóvenes puede ser un indicador de actividad, además de la distribución no generalizada de entesopatías. En el caso de la alteración en las clavículas de I1-S10 e I2-S11, tanto la particularidad del indicador como su aparición a temprana edad nos permite estimar su causa por actividad, por sobre un fenómeno degenerativo.

\section{INDICADORES DE ESTRÉS MECÁNICO EN EL ÁREA SUR ANDINA}

Ejemplos de indicadores de estrés mecánico se han discutido previamente en el área sur andina. A partir de los hallazgos en el cementerio de Maitas del periodo medio de Arica, se asoció la presencia de OV cervical con actividades que implican estrés mecánico por carga de peso. En dicho trabajo la osteofitosis cervical en adultos jóvenes mayoritariamente femeninos fue relacionada con el uso de una mochila para carga o capacho $^{3}$ (Allison et al. 1984: 525-527). En el estudio de Gerszten y Allison (2001) nuevamente se observó una prevalencia de hasta un 30\% de lesiones lumbares y cervicales a partir de una muestra de 700 individuos prehispánicos de los valles de Arica (Chile) e Ica (Perú). La mayor prevalencia de OV cervical en mujeres se interpretó como un marcador ocupacional por el traslado de carga y de lactantes. Lo anterior es reforzado por el hallazgo de una mujer enterrada junto con su capacho, en la cual se reconocieron OA considerable a nivel cervical (Gerszten y Allison 2001). El uso de este medio de transporte también afectaría a las vértebras lumbares, pues como lo destacan Calce et al. (2018) y Cheverko y Bartelink (2017) son el segmento más sensibles a la carga de peso.

Para el área de San Pedro de Atacama, Arriaza (1991) sugirió que las condiciones osteoartríticas observadas en la articulación de la rodilla en la colección de Coyo Oriente podrían ser el resultado de actividades de carga. Para la misma área en colecciones del Periodo Medio y el Intermedio Tardío, LópezBarrales et al. (2015) proponen que la intensidad de la osteofitosis con énfasis en el segmento cervical, se

3/ El capacho es un tipo de bolso que se lleva en la espalda y se sostiene por una correa que se apoya en la frente, dejando libres las manos para su utilización en tareas diferentes al transporte de carga o complementarias con éste (Ballester y Clarot, 2014; López-Barrales et al., 2015). 
relaciona al transporte de carga sobre la cabeza o utilizando ésta como soporte. También proponen que la osteofitosis vertebral severa daría cuenta del uso de capacho en actividades específicas como la minería. Patrones similares fueron observados por Quevedo (2000) en Punta Teatinos, y Ballester y Clarot (2014) en Tocopilla.

Dentro de los pocos antecedentes en el valle de Copiapó, se han registrado lesiones similares en Caserones 61, donde el único entierro adscrito al PT correspondiente a una mujer de alrededor de 23 años que presentó OV en entre L1 y L4 (incluyendo el desarrollo de hernias) y OA en T4 (Rodríguez y Pizarro, 2015). La presencia de hernias, permite estimar que las lesiones tienen relación con la carga de peso, aunque su distribución también puede sugerir lesiones por flexión (similar a lo observado en I1-S10, figura 14). Esta mujer también presentó inserciones musculares marcadas en clavículas y fémures (Rodríguez y Pizarro, 2015). Un segundo caso es el hallazgo en el centro de la ciudad de Copiapó, también adscrito al PT, de una mujer de entre 21 y 26 años que presentó una OA en la patela izquierda (Cabello et al., 2010; Campano, 2010). Ambos casos destacan por tratarse de individuos femeninos jóvenes, con lesiones severas, similares a lo observado en I1-S10 y en I2-S11.

A partir de estos antecedentes, en Iglesia Colorada es posible proponer que las lesiones a nivel cervical y lumbar, junto a las artropatías en rodillas en individuos jóvenes, forman un patrón asociado a actividades frecuentes que implican la carga de peso.

\section{CONCLUSIONES}

El análisis de la colección bioantropológica de Iglesia Colorada se vio considerablemente limitado por el mal estado de preservación de gran parte de la muestra, principalmente de aquellos restos humanos excavados en las campañas de 1995 y 2003. Sin embargo, fue posible reconocer el perfil etario de la población que consiste en un $29 \%$ de nonato-lactantes, un $24.8 \%$ de infantes y juveniles, y un $45,2 \%$ de adultos. En la mayoría de los adultos no se pudo especificar su rango etario y solo dos individuos superaron los 45 años de edad.

Los indicadores dentales sugieren una dieta blanda, posiblemente agrícola, con bajo desgaste dental (Eshed et al. 2004; Fields et al. 2009; Molnar et al. 1972; Smith 1984). Destaca la baja presencia de caries en comparación con muestras contemporáneas asociadas a la cultura Diaguita (Ericksen 1969; Araya-Rosado y Vernacchio-Wilson 2006). Lo anterior amerita más estudios enfocados a la dieta de esta población. La alta pérdida dental observada en el cráneo 2 de la sepultura 19 sugiere un estrés diferencial y que posiblemente las cabezas enterradas aisladas hayan sido de individuos seleccionados por condiciones especiales (Garrido y Morales 2019).

Respecto del perfil paleopatologico, los indicadores de estrés nutricional o de trastornos metabólicos sólo se registraron en adultos y no se pudo establecer si hubo diferencias significativas entre los individuos cuyas cabezas fueron cercenadas y aquellos que fueron enterrados completos debido al tamaño de la muestra. Sin embargo, se reconoció estrés fisiológico en ambos grupos y en el caso de las cabezas aisladas, estos indicadores se presentaron en todos los casos observables (Garrido y Morales 2019). En general, estos indicadores no se presentan de modo severo, dando cuenta de una población sin indicadores atípicos de estrés fisiológico durante su etapa de crecimiento y desarrollo.

En el patrón artropático de Iglesia Colorada, destaca la presencia de lesiones a temprana edad, lo que sustenta su interpretación como indicadores de actividad. El patrón de lesiones cervical y lumbar se observó en hombres, mientras, el único individuo femenino, presentó lesiones en el área lumbar y torácica, junto a 
la OA en hallux. Esto podrían ser reflejo de una diferenciación por sexo. Sin embargo, el reducido número de casos impide comprobar esta hipótesis.

Por el momento sólo es posible afirmar que los individuos adultos de Iglesia Colorada presentan indicadores de actividades de carga de peso por periodos prolongados, donde posiblemente usaron la cabeza como punto de apoyo afectando las vértebras cervicales. También es posible reconocer la presencia de lesiones específicas como la OA bilateral en clavículas y en los pies que podrían estar relacionadas con actividades de molienda o chancado. Este conjunto de indicadores se podría asociar a los antecedentes de explotación minera y agrícola de la zona, incluyendo el posible uso de capachos.

Con respecto a las diferencias cronológicas entre las sepulturas asociadas al PT y la sepultura 10 asociada al PIT, no se observaron diferencias significativas en ninguno de los indicadores revisados. Es más, la coincidencia de estos podría interpretarse como una continuidad en el modo de vida de esta población.

El presente trabajo ofrece la revisión de una de las colecciones más completas del periodo Tardío del valle de Copiapó, y abre una base de comparación que permita abordar la presencia de patologías e indicadores de actividad en la secuencia cronológica de la región. Nuevos análisis interregionales y diacrónicos ayudarán a comprender mejor la especificidad de los cambios biológicos en las comunidades locales del valle introducidos en época Inca y ahondar en la discusión sobre la economía y la distribución del trabajo bajo el fenómeno imperial.

\section{AGRADECIMIENTOS}

Este estudio se enmarca dentro del proyecto Fondecyt 11170010, titulado "Dinámicas sociales en provincias imperiales: expansión Inca y respuesta de agentes locales en el Valle Copiapó". Se agradece el financiamiento a la investigación entregado por la Agencia Nacional de Investigación (ANID), además del apoyo y auspicio del Museo Regional de Atacama (Copiapó) y el Museo Nacional de Historia Natural (Santiago).

\section{REFERENCIAS BIBLIOGRÁFICAS}

ACSADI, G. y J. NEMESKERI. 1970. History of Human Lifespan and Mortality. Akadémiai Kiadó, Budapest.

ALLISON, M.J. 1984.Paleopathology in Peruvian and Chilean populations. En Paleopathology at the Origins of Agriculture, editado por M. N. Cohen y G. J. Armelagos, pp. 515-529. University Press of Florida, Orlando.

ALSUP, B.K. 2007. Investigation of Second, Fourth, and Eighth Sternal Rib End Variation Related to Age Estimation. Master's Thesis. University of Tennessee, Knoxville.

ANDRADE, P. 2012. Análisis Comparativo de Paleopatología Dental de las Poblaciones Prehistóricas del Norte Semiárido de Chile: Una Aproximación Bioarqueológica. Tesis para optar al título de Antropólogo Físico, Universidad de Chile.

ANGEL, L. 1966. Porotic Hyperostosis, Anemias, Malarias, and Marshes in the Prehistoric Eastern Mediterranean. Science 153(3737):760-763.

ARAYA-ROSADO, M., y J. VERNACCHIO-WILSON. 2006. Paleopathology and osteobiography of the people of Peñuelas, Chile's semiarid north. Memórias Do Instituto Oswaldo Cruz 101(2): 85-95.

AUFDERHEIDE, C. y C. RODRIGUEZ-MARTIN. 2006. The Cambridge encyclopedia of human paleopathology, Cambridge University Press, Cambridge.

BALlester, B. y A. CLAROT. 2014. La Gente de los Túmulos de Tierra. Estudio, Conservación y Difusión de Colecciones Arqueológicas de la Comuna de Mejillones. Ilustre Municipalidad de Mejillones, Mejillones.

BASS, W. 2005. Human Osteology: A laboratory and field manual. Missouri Archaeological Society, Columbia.

BECKETT, S. y N.C. LOVELL. 1994. Dental disease evidence for agricultural intensification in the Nubian C-Group. International Journal of Osteoarchaeology 4(3): 223-239. 
BERENGUER, J. 2009. Chile bajo el imperio de los Inkas. MCHAP, Santiago.

BRIDGES, P. 1992. Prehistoric Arthritis in the Americas. Annual Review of Anthropology 21(1): 67-91.

BROOKS, S. y J.M. SUCHEY. 1990. Skeletal Age Determination based on the os pubis: a Comparison of the AcsadiNemeskeri and Suchey-Brooks Methods. Human Evolution, 5(3): 227-238.

BUCKBERRY, J. L.L. y A. T.T. CHAMBERLAIN. 2002. Age estimation from the auricular surface of the ilium: A revised method. American Journal of Physical Anthropology 119(3):231-39.

BUCHET L. 1989. L'usage de la station accroupie dans les sociétés antiques et médiévales de Gaule. Actes temes Journées Anthropologiques, pp.114-122. Centre National de la Recherche Scientifique, Paris.

BUIKSTRA, J. y D.H. UBELAKER. 1994. Standards for data collection from human skeletal remains. Archeological Survey Research Series. Fayetteville, Arkansas.

BURT, N.M., D. SEMPLE, K. WATERHOUSE y N.C. LOVELL. 2013. Identification and Interpretation of Joint Disease in Paleopathology and Forensic Anthropology. Charles C. Thomas, Springfield, llinois.

CALCE, S.E., H.K. KURKI, D.A WESTON y L. GOULD. 2018. The relationship of age, activity, and body size on osteoarthritis in weight- bearing skeletal regions. International Journal of Paleopathology 22: 45-53.

CASTILLO, G. 1998. Los períodos intermedio tardío y tardío: desde la cultura Copiapó al dominio Inca. En Culturas Prehistóricas de Copiapó, editado por M. Cervellino, G. Castillo y H. Niemeyer, pp. 168-282. Museo Regional de Atacama, Copiapó.

CHEVERKO, C. M. y E.J. BARTELINK. 2017. Resource intensification and osteoarthritis patterns: changes in activity in the prehistoric Sacramento-San Joaquin Delta region. American Journal of Physical Anthropology 164(2): 331-342.

COSTA-JUNQUEIRA, M. A., H. VARELA, J. COCILOVO, S. QUEVEDO y S. VALDANO. 2000. Perfil paleodemográfico de Morro de Arica, una población de pescadores arcaicos del Norte de Chile. Boletín Del Museo Nacional de Historia Natural 49: 215-235.

CUCINA, A. 2011. Manual de Antropologia Dental. Ediciones de la Universidad Autónoma de Yucatán, Mérida.

DÍAZ, P. y A. PACHECO. 2017. Informe de las dataciones radiocarbónicas y de los análisis de isótopos estables (13C, $15 \mathrm{~N}$ y $18 \mathrm{O})$ de los contextos funerarios del proyecto Caserones. Informe inédito.

EL-NAJJAR, M.Y., D.J. RYAN, C. TURNER y B. LOZOFF. 1976. The etiology of porotic hyperostosis among the prehistoric and historic Anasazi Indians of Southwestern United States. American Journal of Physical Anthropology 44(3): 477-487.

ESHED, V., A. GOPHER, T.B. GAGE y I. HERSHKOVITZ. 2004. Has the transition to agriculture reshaped the demographic structure of prehistoric populations? New evidence from the Levant. American Journal of Physical Anthropology 124(4): 315-329.

ERICKSEN, M. 1969. Los habitantes Pre-colombinos del Norte Chico: una síntesis de trabajo en progreso. Boletín del Museo Nacional de Historia Natural 30: 319-337.

ERICKSEN, M. 1978. Restos Óseos de Punta Piedra. Boletín Del Museo Arqueológico de La Serena 16: 189-210.

FIELDS, M., E. HERSCHAFT, D.L. MARTIN y J.T. WATSON. 2009. Sex and the agricultural transition: Dental health of early farming females. Journal of Dentistry and Oral Hygiene 1(4): 42-51.

FINNEGAN, M. 1978. Non-metric variation of the infracranial skeleton. Journal of Anatomy 125(1): 23-37.

GARRIDO, F. 2016. Rethinking Imperial Infrastructure: A Bottom-up Up Perspective on the Inca Road. Journal of Anthropological Archaeology 43:94-109.

GARRIDO, F. y C. MORALES. 2019. Displays of Violence and Power at the Edge of the Empire: Provincial Trophy Heads during Inca Times. Latin American Antiquity 30(3): 606-623.

GARRIDO, F. y M.T. PLAZA. 2020. Provincial Inca metallurgy in northern Chile: New data for the Viña del Cerro smelting site. Journal of Archaeological Science: Reports 33(October):1-13.

GENOVES, S. 1967. Proportionality of the Long Bones and their Relationship Stature among Mesoamericans. American Journal of Physical Anthropology 26: 67-78.

GERSZTEN, E. y M.J. ALLISON. 2001. Diseases of the Spine in South American Mummies. Neurosurgery 48(1): 208-213.

GONZALEZ, P. 2013. Arte y cultura diaguita chilena: simetría, simbolismo e identidad. SCHA, Ucayali editores, Santiago. 
GOODMAN, A. y A.H. GOODMAN. 1988. The Chronology Of Enamel Hypoplasias In An Industrial Population A reappraisal of Sarnat and Shour 1941 1942. Human Biology, 60(5), 781-791.

GOODMAN, A. H. y J.C. ROSE. 1991. Dental Enamel Hypoplasias as Indicators of Nutritional Status. Advances in Dental Anthropology, 279-293.

GOYCOOlEA, M. V., M. CAStro, M. GAlVez, C. MONTOYA, J. FUENTES y V. Silva-Pinto. 2019. Otitis media and mastoiditis in temporal bones of prehistoric Chilean populations. A paleopathological and paleoepidemiological study. Acta Oto-Laryngologica 139(4):340-344.

HILLSON, S. 1996. Dental Anthropology. Cambridge University Press, Cambridge.

HUARD P. y M. MONTAGNÉ. 1951. Le squelette humain et l'attitude accroupie. Bulletin de la Societè des Etudes Indochinoises 26: 401-426.

IRISH, J.D., SCOTT, G.R. y R. Scott. 2015. A Companion to Dental Anthropology. John Wiley y Sons, Ltd, Chichester. IRIBARREN, J. 1958. Arqueología en el valle de Copiapó. Revista Universitaria 43(22):167-195.

JURMAIN, R. D. y L. KILGORE. 1995. Skeletal evidence of osteoarthritis: A palaeopathological perspective. Annals of the Rheumatic Diseases 54(6): 443-450.

KATZENBERG, M. A., D. A. HERRING, y S.R. SAUNDERS. 1996. Weaning and infant mortality: Evaluating the skeletal evidence. American Journal of Physical Anthropology 101(S23):177-99.

KEISER-NIELSEN S. 1971. Two-Digit System of Designating Teeth. Dental Practice (Ewell) 3(4):6.

KOCHER, M., R. TUCKER, T.J. GANLEY y J.M. FLYNN. 2006.Management of Osteochondritis Dissecans of the Knee: Current Concepts Review. The American Journal of Sports Medicine 34(7):1181-1191.

KOTHARI, A., P. PONCE, B. ARRIAZA y L. O'CONNOR-READ. 2009. Osteochondritis dissecans of the knee in a mummy from Northern Chile. Knee 16(2):159-60.

KNÜSEL, C., J. S. GÖGGEL y D. LUCY. 1997. Comparative degenerative joint disease of the vertebral column in the medieval monastic cemetery of the gilbertine priory of St. Andrew, Fishergate, York, England. American Journal of Physical Anthropology 103(4):481-95.

LAI, P. y N.C. LOVELL. 1992. Skeletal Markers of Occupational Stress in the Fur Trade : a Case Study from a Hudson ’s Bay Company Fur Trade Post. International Journal of Osteoarchaeology, 2(i 992), 221-234.

LALLO, J., G. ARMELAGOS y R. MENSFORTH. 1977. The Role of Diet, Disease, and Physiology in the Origin of Porotic Hyperostosis. Human Biology 49(3):471-483.

LEWIS, M. 2007. The Bioarchaeology of Children: Perspectives from Biological and Forensic Anthropology. Cambridge University Press, Cambridge.

LÓPEZ-BARRALES, R., M. HUBBE, A. EUGENIO, W.A NEVES y H.M. NIEMEYER. 2015. Osteofitosis vertebral en poblaciones prehispánicas de San Pedro de Atacama, norte de Chile. Estudios Atacameños Arqueología y Antropología Surandinas 50: 177-194.

LOUDON, J. K. 2016. Original research biomechanics and pathomechanics. The International Journal of Sports Physical Therapy 11(6): 820-830.

LUKACS, J. R. y L. LARGAESPADA. 2006. Explaining sex differences in dental caries prevalence: Saliva, hormones, and "life history" etiologies. American Journal of Human Biology 18(4): 540-555.

MANN, R. W., R. L. JANTZ, W. M. BASS y P.S. WILLEY. 1991. Maxillary Suture Obliteration: A Visual Method for Estimating Skeletal Age. Journal of Forensic Sciences, 36(3): 781-791.

MARIOTTI, V, F. FACCHINI y M. G. BELCASTRO. 2004. Enthesopathies - proposal of a standardised scoring method and applications. Collegium antropologicum 28(1):145-59.

MASSLER, M., I. SCHOUR y H.G. PONCHER. 1941. Developmental pattern of the child as reflected in the calcification pattern of the teeth. Archives of Pediatrics y Adolescent Medicine 62(1): 33.

MAYS, S.A. 2005. Paleopathological study of hallux valgus. American Journal of Physical Anthropology 126(2): 139-149.

MIQUEL-FEUCHT, M. J., M. POLO-CERDÁ y J.D. VILLALAÍN-BLANCO. 1999. El síndrome criboso: criba femoral vs criba orbitaria. Paleopatología, Actas Actas V Congreso Nacional AEP, Alcalá la Real pp.221-237.

MOLNAR, S. 1971.Human tooth wear, tooth function and cultural variability. American Journal of Physical Anthropology 34(2): 175-189. 
MOLNAR, S., M.J. BARRETT, L. BRIAN, C.L. BRACE, D.S. BROSE, J.R. DEWEY y G.A. WRIGHT. 1972. Tooth Wear and Culture: A Survey of Tooth Functions among Some Prehistoric Populations. Current Anthropology 13(5): 511-526.

MOLlESON, T. 1994. The eloquent bones of Abu Hureyra. Scientific American 271(2):70-75.

MUNIZAGA, J. 1973. Síntesis de la Antropología Física del Norte Chico. Actas Del VI Congreso de Arqueología Chilena Boletín de Prehistoria, Número Especial., 345-351.

NIEMEYER, H. 1998. El periodo Medio. Complejo Las Ánimas. En Culturas Prehistóricas de Copiapó, editado por M. Cervellino, G. Castillo y H. Niemeyer, pp. 115-162. Museo Regional de Atacama, Copiapó.

NIX, S., M. SMITH y B. VICENZINO. 2010. Prevalence of hallux valgus in the general population : a systematic review and meta- analysis. Journal of Foot and Ankle Research 3(1):21

ORTNER, D. 2003. Identification of pathological conditions in human skeletal remains. Elsevier Academic Press, San Diego.

PACHECO, A. 2016. Informe Bioarqueológico de los contextos mortuorios recuperados durante el Rescate de Caserones 61 (Comuna de Tierra Amarilla, Provincia de Copiapó; III Región). Informe inédito

PECHENKINA, E., R. BENFER y W. ZHIJUN. 2002. Diet and health changes at the end of the Chinese Neolithic: The Yangshao/Longshan transition in Shaanxi province. American Journal of Physical Anthropology 117(1): 15-36.

PHENICE, T.W. 1969. A newly developed visual method of sexing the os pubis. Americal Journal of Physical Anthropology 30: 297-301.

PINHASI, R. y S. MAYS. 2007. Advances in Human Palaeopathology. John Wiley y Sons, Ltd, Chichester.

QUEVEDO, S. 2000. Patrones de actividad a través de las patologías en población arcaica de Punta Teatinos, norte semiárido Chileno. Chungará 32(1): 7-9.

RAI, V., S. SAHA, G. YADAV, A.M. TRIPATHI y K. GROVER. 2014. Dental and skeletal maturity- A biological indicator of chronologic age. Journal of Clinical and Diagnostic Research 8(9): 60-64.

RESNICK, D. 2002. Degenerative disease of the spine. En: D. Resnick (Ed.), Diagnosis of bone and joint disorders, Vol 2 (4th ed.) (pp. 1382-1485). Philadelphia: Saunders.

ROBB, J. 1994. Skeletal signs of activity in the Italian metal ages: Methodological and interpretative notes. Human Evolution 9(3): 215-229.

ROBB, J. 1998. The Interpretation of Skeletal Muscle Sites: A Statistical Approach. International Journal of Osteoarchaeology 8(5):363-77.

ROBERTS, C. y K. MANCHESTER. 2010. The Archaeology of Disease. History Press, Gloucestershire.

RODRÍGUEZ M., y M. PIZARRO. 2015. Informe Bioantropológico Proyecto Caserones: Analisis E25 y 29 Sitio Caserones 61. Informe inédito.

ROJAS-SEPÚLVEDA, C.M. y O. DUTOUR. 2014 Enfermedad articular degenerativa en seis colecciones prehispánicas del noroccidente de Amárica del Sur. Chungara Revista de Antropología Chilena, 46(1), $153-169$.

ROTHSCHILD, B. 2012. Extirpolation of the Mythology that Porotic Hyperostosis is caused by Iron Deficiency Secondary to Dietary Shift to Maize. Advances in Anthropology 2(3):157-160.

ROTHSCHILD, B.M. y R.J. WOODS. 2012. Epidemiology and Biomechanics of Osteoarthritis. Principles of Osteoarthritis- Its Definition, Character, Derivation and Modality-Related Recognition, 590. https://doi. org/10.5772/28463

RUDE, R. 1998. Magnesium deficiency: a cause of heterogeneous disease in humans. Journal of Bone and Mineral Research 13(4): 749-458.

SAYER, D. y S.D. DICKINSON. 2013. Reconsidering obstetric death and female fertility in Anglo-Saxon England. World Archaeology, 45(2):285-97.

SCHAEFER, M., S. BLACK y L. SCHEUER. 2009. Juvenile Osteology. Juvenile Osteology. Elsevier Academic Press, Burlington.

SCHEUER, L. y S. BLACK. 2004. The Juvenile Skeleton. Elsevier Academic Press, Londres.

SCHULTZ, M. 2001. Paleohistopathology of bone: A new approach to the study of ancient diseases. American Journal of Physical Anthropology, 116(33): 106-147. 
SMITH, B.H. 1984. Patterns of Molar Wear in Hunter- Gatherers and Agriculturalists. American Journal of Physical Anthropology 63(1): 39-56.

STEWART T.D. 1958. The Rate of Development of Vertebral Osteoarthritis in American Whites and Its Significance in Skeletal Age Identification. The Leech 28:144-151.

STEWART T.D. 1966. Some Problems in Human Palaeopathology. En Human Palaeopathology, editado por S. Jarcho, pp.43-55. Yale University Press, New Haven.

STUART-MACADAM, P. 1989. Nutritional Deficiency Diseases: A Survey of Scurvy, Rickets and Iron-deficiency Deficiency Anemia. En Reconstruction of Life from the Skeleton, editado por Yasar Iscan and Kenneth Kennedy, pp. 201--222. Liss, New York.

STUART-MACADAM. 1992. Anemia in past populations. En Demography and Disease: Changing Perspectives of Anemia P. Stuart-Macadam y S. Kent Diet, pp.151-170. Aldine de Gruyter, New York.

SZILVÁSSY, J. 1988. Altersdiagnose am Skelett [Age estimation of the skeleton] En Anthropologie, editado por R. Knussmann, pp.421-435. Handbuch der vergleichenden Biologie des Menschen. Fischer Verlag, Stuttgart.

TRONCOSO, A. y D. PAVLOVIC. 2013.Historia, saberes y prácticas: Un ensayo sobre el desarrollo de las comunidades alfareras del norte semiárido chileno. Revista Chilena de Antropología 27: 101-140.

UBELAKER, D.H. 1979. Skeletal evidence for kneeling in prehistoric Ecuador. American Journal of Physical Anthropology 51: 679-685.

URIBE, M. y R. SÁNCHEZ. 2016. Los Incas en Chile. Aportes de la arqueología chilena a la historia del Tawantinsuyu. Prehistoria en Chile. Desde sus primeros habitantes hasta los Incas, F. Falabella, M. Uribe, L. Sanhueza, C. Aldunate y J. Hidalgo (eds.). Ed. Universitaria, Santiago, pp.529-572.

VILLOTTE, S., S. ASSIS, F. ALVES CARDOSO, C. YVETTE HENDERSON, V. MARIOTTI, M. MILELLA, D. PANY-KUCERA, N. SPEITH, C.A. WILCZAK y R. JURMAIN. 2016. In search of consensus: Terminology for entheseal changes (EC). International Journal of Paleopathology 13:49-55.

Villotte, S., D. CASTEX, V. COUALlier, O. DUTOUR, C.J. KNÜSEL y D. HENRY-GAMBIER. 2010. Enthesopathies as occupational stress markers: Evidence from the upper limb. American Journal of Physical Anthropology 142(2):224-34.

VOLK, A. y J. ATKINSON. 2013. Infant and child death in the human environment of evolutionary adaptation. Evolution and Human Behavior 34(3):182-92.

WALDRON, T. 2008. Palaeopathology. Cambridge University Press, Cambridge.

WALKER, P. L., J. JOHNSON y P. LAMBERT. 1988. Age and Sex Biases in the Preservation of Human Skeletal Remains. American Journal of Physical Anthropology 76: 183-188.

WEISS, E. 2009. Introduction to Bioarchaeology: History to Present. En Bioarchaeological science : what we have learned from human skeletal remains, pp.9-37. Nova Science Publishers, New York.

WEISS, E., y R. JURMAIN. 2007. Osteoarthritis revisited: a contemporary review of aetiology. International Journal of Osteoarchaeology 17(5): 437-450.

WELLS C. 1962. Joint Pathology in Ancient Anglo-Saxons. Journal of Bone and Joint Surgery 44(B4): 948-949.

WRIGHT, L.E. y H.P. SCHWARCZ. 1998. Stable Carbon and Oxygen Isotopes in Human Tooth Enamel: Identifying Breasatfeeding and Weaning in Prehistory. American journal of physical anthropology, 106, 1-18. 